Supplement of Hydrol. Earth Syst. Sci., 23, 4491-4508, 2019

https://doi.org/10.5194/hess-23-4491-2019-supplement

(c) Author(s) 2019. This work is distributed under

the Creative Commons Attribution 4.0 License.

(c) (1)
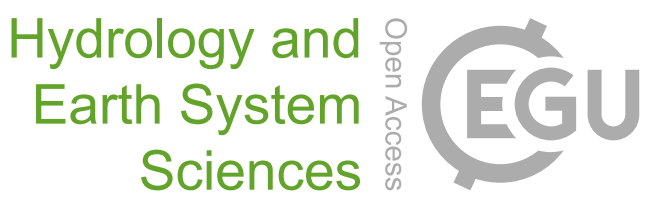

Supplement of

\title{
Assessing the impacts of hydrologic and land use alterations on water temperature in the Farmington River basin in Connecticut
}

John R. Yearsley et al.

Correspondence to: John R. Yearsley (jyearsle@uw.edu)

The copyright of individual parts of the supplement might differ from the CC BY 4.0 License. 


\section{Supplementary Material}

\section{SM.1 State-Space Model Structure}

Mathematical models with state-space structure (Schweppe, 1973) can provide decision support methods for developing management plans that consider the impacts of hydrologic and land use alterations on water temperature. State-space models for continuous time have the general, nonlinear form:

$$
\frac{d C(t)}{d t}=f[C(t), w(t), u(t)]
$$

and discrete time:

$$
C(n \Delta+\Delta)=\phi[C(n \Delta), w(n \Delta), u(n \Delta),(n \Delta)]
$$

where $C()$ is a vector of state variables, $f$ and $\phi$ are known, nonlinear functions, $u$ are known inputs with uncertainty characterized by $w$, and the evolution of time is described by the number of time steps, $n$, at discrete intervals, $\Delta$.

In practice, linearization methods are used to obtain solutions to (2) in the form (Schweppe, 1973):

$$
C(n \Delta+\Delta)=\phi[C(n \Delta), n \Delta]+B[C(n \Delta)] u(n \Delta)+G[x(n \Delta)] w(n \Delta)
$$

where, $B[C(n \Delta)]$ and $u(n \Delta)$ are the known inputs, $w(n \Delta)$ is a zero mean uncertainty process, and $G[x(n \Delta)]$ is a known function of time characterizing the uncertainty,

When a solution exists that is reasonably close to the true system state, $C(n \Delta)$, then (3) can be written, to first order, as:

$$
C^{(1)}(n \Delta+\Delta)=\phi\left[C_{n o m}(n \Delta), n \Delta\right]+B\left[C_{n o m}(n \Delta), n \Delta\right] u(n \Delta)+F^{(1)}\left[C_{n o m}(n \Delta), n \Delta\right]
$$

where $C_{n o m}(n \Delta)$ is a state estimate for which the uncertainty is zero, $(w(n \Delta)=0.0), B\left[C_{n o m}(n \Delta)\right.$, $n \Delta]$ and $u(n \Delta)$ are the known inputs, and $F^{(1)}\left[C_{n o m}(n \Delta), n \Delta\right]$ is a first-order model of the uncertainty. The nominal solution differs conceptually from the so-called "deterministic" solution, for which the uncertainty is also zero. Deterministic solutions consist of a single solution that is fully determined by the parameter values. The nominal solution is only an estimate of the true system state. So long as the nominal solution remains close to the true system state, linearization of the elements of the nominal solution can be used to estimate uncertainty (Schweppe, 1973), as in Yearsley (2012).

\section{SM.2 Stream and River Segments Dominated by Advection}

The linearized, discrete-time nominal $(w(\mathrm{n} \Delta)=0.0)$ solution for a gridded river network in a Lagrangian frame of reference (Yearsley, 2009) having the form of (1) is given by 


$$
\begin{aligned}
& T_{n o m}\left(n \Delta+\Delta\left(x_{j}\right), x_{j}\right)= \\
& T_{n o m}\left(n \Delta, x_{j}\right)+\left\{\frac{F^{T}\left[T_{n o m}\left(n \Delta, x_{j}\right)\right]+h^{T}\left(n \Delta, x_{j}\right)+B^{T}\left[T_{n o m}\left(n \Delta, x_{j}\right)\right] u\left(n \Delta, x_{j}\right)}{\rho C_{p} D\left(n \Delta, x_{j}\right)}\right\} \Delta\left(x_{j}\right)
\end{aligned}
$$

where $T_{\text {nom }}()$ is water temperature $\left({ }^{\circ} \mathrm{C}\right), \rho$ is the density of water $\left(\mathrm{kg} / \mathrm{m}^{3}\right), C_{p}$ is the specific heat capacity of water $\left(\mathrm{J} / \mathrm{kg} /{ }^{\circ} \mathrm{C}\right), F^{T}[]$ is the vector transpose of the thermal energy budget components that are a function of the water temperature $T()\left(\mathrm{W} / \mathrm{m}^{2}\right), h^{T}()$ is the vector transpose of the known inputs to the thermal energy balance that are not a function of the water temperature $T(), D\left(n \Delta, x_{j}\right)$ is channel depth (m) at time $n \Delta$ and distance $x_{j}$, and $B^{T}()$ is the vector transpose of advected sources of thermal energy from tributaries, groundwater point sources and hyporheic flow $\left({ }^{\circ} \mathrm{C} / \mathrm{s}\right)$, and $\Delta\left(x_{j}\right)$ is the time increment for a Lagrangian parcel to travel from the upstream (us) starting point, $x_{j}^{u s}$, to the boundary of the $\mathrm{j}^{\text {th }}$ grid cell at $x_{j}$.

$$
\Delta\left(x_{j}\right)=U(j) /\left(x_{j}^{u s}-x_{j}\right)
$$

where $U(j)$ is the river speed in the $j^{t h}$ grid cell $(\mathrm{m} / \mathrm{s}), x_{j}^{u s}$ is the upstream location of a Lagrangian parcel (meter), $x_{j}$ is the downstream location (in meter) of a Lagrangian parcel after a time of travel $\Delta\left(x_{j}\right)$.

Stream depth, $D\left(n \Delta, x_{j}\right)$, and stream speed, $U\left(n \Delta, x_{j}\right)$, in the particle-tracking algorithm are estimated with the method of Leopold and Maddock (1953), as in Chapra et al. (2003), and Yearsley (2012):

$$
\begin{aligned}
& D\left(n \Delta x, x_{j}\right)=d_{a} Q\left(n \Delta x, x_{j}\right)^{d_{b}} \\
& U\left(n \Delta x, x_{j}\right)=u_{a} Q\left(n \Delta x, x_{j}\right)^{u_{b}}
\end{aligned}
$$

where the parameters, $d_{a}, d_{b}, u_{a}$ and $u_{b}$ are estimated from rating tables prepared by USGS for the freely-flowing stream segments and $Q\left(n \Delta, x_{j}\right)$ is the segment streamflow and from reservoir geometry obtained from the US Army Corps of Engineers' (USACE) (2016) National Inventory of Dams (NID) for impoundment segments.

For those lakes and impounded segments with shallow depths and short residence times we estimated the speed of the water parcel $U\left(n \Delta, x_{j}\right)$, could be estimated from the continuity equation:

$$
U\left(n \Delta, x_{j}\right)=\frac{Q\left(n \Delta, x_{j}\right)}{A_{x}\left(n \Delta, x_{j}\right)}
$$

where $\mathrm{A}_{\mathrm{x}}\left(n \Delta, x_{j}\right)$ is the cross-sectional area of the impoundment segment.

The solution to Equation (5) is subject to the initial conditions at time, $n \Delta=0$, 


$$
T\left[0, x_{j}\right]=T_{0}\left(x_{j}\right)
$$

and the headwaters conditions

$$
T_{\text {head }}\left[n \Delta, x_{\text {head }}\right]=T_{M}\left[n \Delta, x_{\text {head }}\right]
$$

As in previous studies (Sun et al., 2015; Wu et al., 2012; Yearsley, 2012, 2009), we estimated headwaters temperatures with a nonlinear regression of the logistic relationship between air temperature and water temperature due to Mohseni et al. (1998):

$$
T_{\text {head }}=\mu+\frac{\alpha-\mu}{1+e^{\gamma\left(\beta-T_{\text {smooth }}\right)}}
$$

where $T_{\text {head }}$ is daily headwaters temperature $\left({ }^{\circ} \mathrm{C}\right), T_{\text {smooth }}$ is smoothed air temperature $\left({ }^{\circ} \mathrm{C}\right)$, and $\alpha$, $\beta, \mu, \gamma$ are regression parameters.

The terms in Equation (5) for advection and the transfer of thermal energy at the airwater interface due to incoming longwave and shortwave radiation, emitted and reflected radiation from the water surface, and latent and sensible heat transfer resulting from evaporation and conduction and uncertain processes in the thermal energy budget. Parameterization for the elements of $F^{T}()$ and $h^{T}()$ related to the transfer of thermal energy across the air-water interface is based on first principles of physics and atmospheric chemistry as well as numerous field studies (e.g., Tennessee Valley Authority, 1972; Boyd and Kasper, 2003). Elements of the matrices, $F^{T}($ ) and $h^{T}()$, are defined below. However, it is necessary to estimate other elements of the parameter space independently. This includes the relationships for initial conditions (Equation 7), the headwaters temperatures (Equation 8), and those that determine the stream hydraulic response, as characterized by stream speed and depth.

For these assumptions, the nominal solutions to the thermal energy budget for those stream and river segments that we have assumed will stratify vertically are:

\section{Epilimnion}

$$
T_{e p i}(n \Delta+\Delta)=T_{e p i}(n \Delta)+\left\{\frac{F\left[T_{e p i}(n \Delta)\right]+h_{e p i}(n \Delta)}{\rho C_{p} V_{e p i}}+\frac{B\left(T_{e p i}(n \Delta)\right)}{V_{e p i}} u\left(T_{e p i}(n \Delta)\right)\right\} A_{e p i} \Delta
$$

Hypolimnion

$$
T_{h y p}(n \Delta+\Delta)=T_{h y p}(n \Delta)+\left\{\frac{B\left(T_{h y p}(n \Delta)\right)}{V_{h y p}} u\left(T_{h y p}(n \Delta)\right)\right\} A_{h y p} \Delta
$$

Elements of $F\left[T_{e p i}()\right], h_{e p i}()$ and $B\left(T_{h y p}()\right)$ are defined below.

The thermal energy budget for each stream segment is comprised of the thermal energy that is transferred across the air-water interface and the advected energy from tributary inflow. 
The formulation of the elements in the terms, $F[T(n \Delta)]$ and $h(n \Delta)$, of Equations (5), (9a) and (9b) is derived from previous studies (Boyd and Kasper, 2007; WEBB and ZHANG, 1997) and from the MTCLIM algorithms developed for purposes of forcing large scale hydrologic models (Bohn et al., 2013; Thornton and Running, 1999):

$$
F[T(n \Delta)]=H_{c o n}[T(n \Delta)]-H_{b a c k}[T(n \Delta)]-H_{\text {evap }}[T(n \Delta)]
$$

where $H_{c o n}[T(n \Delta)]$ is thermal energy flux due to conduction, $H_{b a c k}[T(n \Delta)]$ is the flux due to black body radiation from the water surface, and $H_{\text {evap }}[T(n \Delta)]$ is the flux due to the latent heat loss due to evaporation.

$$
h(n \Delta)=H_{s w} T(n \Delta)+H_{l w} T(n \Delta)
$$

where $H_{s w} T(n \Delta)$ is the shortwave solar radiation reaching the water surface after attenuation by riparian vegetation and $H_{l w} T(n \Delta)$ is the longwave atmospheric radiation reaching the water surface.

The advected thermal energy, $B[T(n \Delta)]$, is associated with tributary inflow to the stream segment;

$$
B[T(n \Delta)]=S_{\text {mix }}(n \Delta)\left[T_{\text {trib }}(n \Delta)-T(n \Delta)\right]
$$

where $S_{\text {mix }}(\mathrm{n} \Delta)$ is the ratio of the volume of the tributary inflow to the segment volume and $\mathrm{T}_{\text {trib }}(\mathrm{n} \Delta)$ water temperature of the tributary inflow.

\section{SM.3 Stream Temperature Model Evaluation}



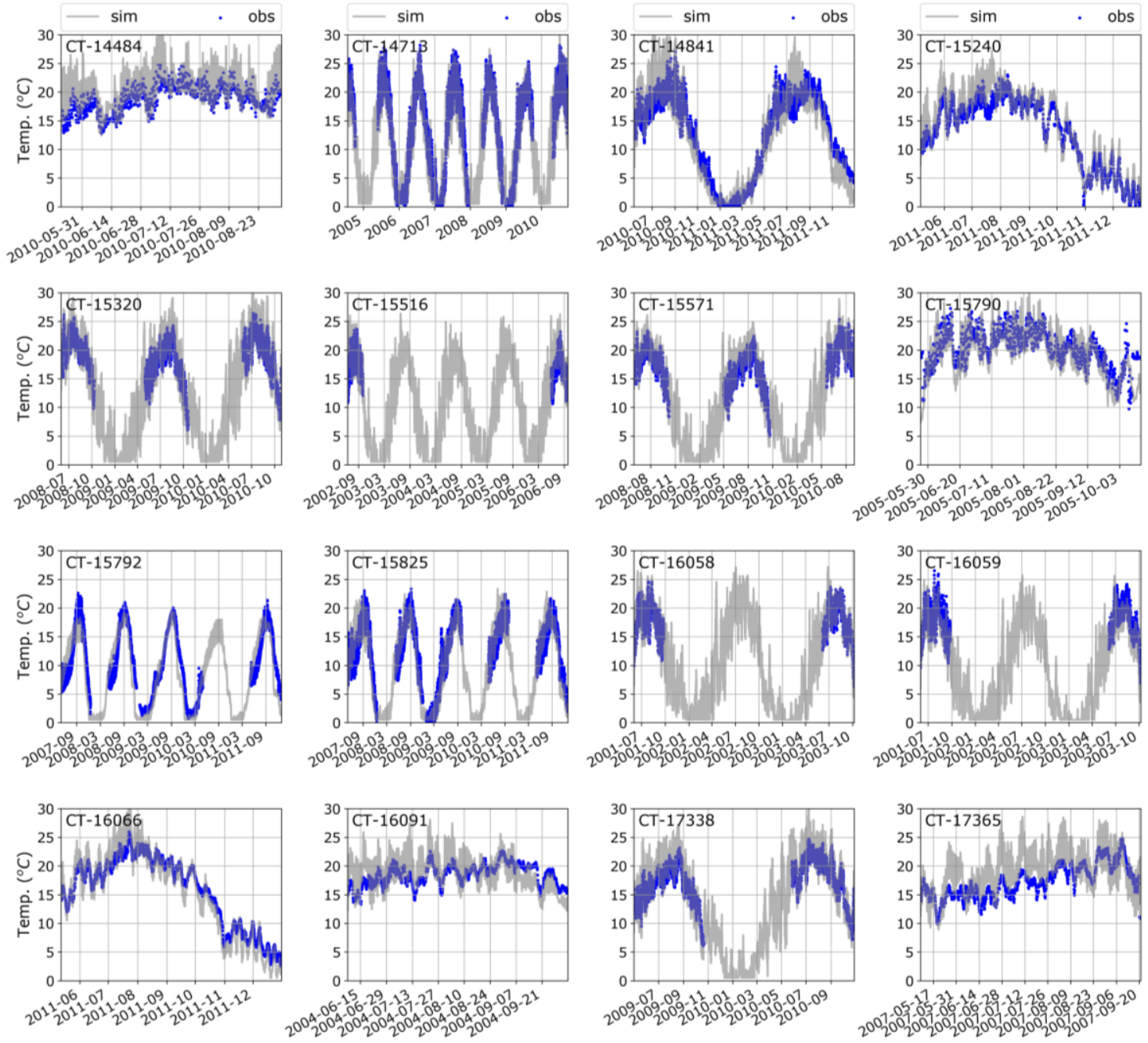

Figure S.1: Time series comparing simulated and observed three-hourly stream temperatures at 16 CTDEEP sites in the Farmington River basin. 